\title{
Sliding Mode Control for Mass Moment Aerospace Vehicles Using Dynamic Inversion Approach
}

\author{
Xiao-Yu Zhang, Yu-Xin Zhao, De-Xin Xu, and Kun-Peng He \\ College of Automation, Harbin Engineering University, Harbin 150001, China \\ Correspondence should be addressed to Xiao-Yu Zhang; zhangxiaoyu@hrbeu.edu.cn
}

Received 26 July 2013; Accepted 28 August 2013

Academic Editor: Rongni Yang

Copyright ( 2013 Xiao-Yu Zhang et al. This is an open access article distributed under the Creative Commons Attribution License, which permits unrestricted use, distribution, and reproduction in any medium, provided the original work is properly cited.

\begin{abstract}
The moving mass actuation technique offers significant advantages over conventional aerodynamic control surfaces and reaction control systems, because the actuators are contained entirely within the airframe geometrical envelope. Modeling, control, and simulation of Mass Moment Aerospace Vehicles (MMAV) utilizing moving mass actuators are discussed. Dynamics of the MMAV are separated into two parts on the basis of the two time-scale separation theory: the dynamics of fast state and the dynamics of slow state. And then, in order to restrain the system chattering and keep the track performance of the system by considering aerodynamic parameter perturbation, the flight control system is designed for the two subsystems, respectively, utilizing fuzzy sliding mode control approach. The simulation results describe the effectiveness of the proposed autopilot design approach. Meanwhile, the chattering phenomenon that frequently appears in the conventional variable structure systems is also eliminated without deteriorating the system robustness.
\end{abstract}

\section{Introduction}

Some of the earliest flight vehicles are controlled by moving the body of the pilot to affect the center of mass (c. m.) of the vehicle. The change in vehicle center of mass alters the relative location of the center of mass with respect to the external forces, thereby effecting a change in the vehicle's motion. Recently, mass movement has been proposed as a control methodology for flight vehicles in atmospheric and exoatmospheric engagements. Advances in aerodynamics subsequently made the moving-mass approach to flight control obsolete in all, but a few, specialized applications. In recent years, techniques of controlling the flight of missiles have gravitated to systems that deliver relatively large amounts of control authority. Several studies have suggested that Mass Moment Control System (MMCS) appears to offer the greater design and cost advantages [1-6], such as controlling the missiles at extreme Mach number and meeting the need for maneuverability and agility. The MMCS changes the vehicle center of mass relative to the external forces to generate the desired control moments. For instance, if the thrust is aligned with the vehicle longitudinal body axis containing the nominal center of mass, moving the center of mass off the body centerline will result in thrust moments about the pitch-yaw axes. Additionally, roll moments will be generated if the thrust or drag has an angular misalignment with respect to the longitudinal axis, or if the vehicle is subject to an aerodynamic lift force. There are some advantages of mass moment control as follows [7, 8]: (1) all the mechanism of MMCS is in aerospace vehicles, which will not affect the aerodynamic configuration and is better to accuracy of the terminal attack. (2) The actuators of MMCS are internal moving masses, which decrease the thermal load of aerodynamic configuration and avoid the gap on the surface of the vehicle and ablation steering surface. (3) By using aerodynamic forces generated by high-speed flight of vehicle, we can decrease the energy consumption and get effective control avoiding the conflict between fuel consumption and control moment generated by lateral jet engine.

Sliding mode control is a robust control technique which has many attractive features such as robustness to parameter variations and insensitivity to disturbances [9-12]. The sliding mode controller is composed of an equivalent control part that describes the behavior of the system when 
the trajectories stay over the sliding manifold and a variable structure control part that enforces the trajectories to reach the sliding manifold and presents them leaving the sliding manifold. Sliding mode control is one of the best choices for controlling perturbed systems with time-delay [13-15]. The price for achieving the robustness/insensitivity to these disturbances is control chattering. The traditional ways for reducing chattering are as follows: (a) replacing the discontinuous control function by "saturation" or "sigmoid ones" $[16,17]$. This approach yields continuous control and chattering elimination. However, it constrains the sliding system's trajectories not to the sliding surface but to its vicinity losing the robustness to the disturbances. (b) Using the higher-order sliding mode control techniques [18-24]. This approach allows driving the sliding variable to zero and its consecutive derivatives in the presence of the disturbances/uncertainties increasing the accuracy of the sliding variable stabilization, and has still been successfully applied for the control of electropneumatic actuators $[25,26]$. Nonetheless, the main challenge of high-order sliding mode controllers is the use of high-order time derivatives of the sliding variable. It is worth noting that some second-order sliding mode control, the popular super-twisting algorithm [27] and gain-commuted controller [28], only require measurement of the sliding variable; whereas the other secondorder sliding mode controllers also need the time derivative of the sliding variable. (c) Using controllers with dynamical gains. Recently, adaptive sliding mode controllers have been proposed, with the interest being the adaptation of the gain magnitude with respect to uncertainty/perturbation effects. Then, a reduced gain induces lower chattering. In [29], an adaptive (first order) sliding mode controller has been proposed and has been evaluated for the control of an electropneumatic actuator. (d) Another technique is the use of fuzzy sliding mode control $[30,31]$. The main advantage of the method is that the performance of the system is improved in the sense of removing the chattering in comparison with the same SMC technique without using fuzzy logic algorithm, and the robust behavior of the system cannot be deteriorated.

Considering the above-mentioned issues, in this paper, we investigate the control of MMAV using FSMC based on dynamic inversion approach. This paper is organized as follows. In Section 2, the mathematical model of MMAV is presented. Based on dynamic inversion, Section 3 gives the main methodological results concerning the fuzzy sliding mode control algorithm. In Section 4, simulation demonstrates the ability of the controller to effectively control the MMAV's motion. Conclusion is given in Section 5.

\section{Mass Moment Aerospace Vehicles Model}

2.1. The General Dynamical Model of the MMAV. The basic principle by which an MMCS is able to control the vehicle's motion is to produce the control torque by using the aerodynamic forces and moving the masses within the MMAV to offset the c. m. of system.

Supposing that the MMAV includes $n$ moving masses and the mass of MMAV's shell are $m_{B}$. The mass of the $i$ th moving mass is $m_{i}$. So, the total mass of MMAV is $m_{C}=m_{B}+\sum_{i=1}^{n} m_{i}$. The mass ratio of the $i$ th moving mass is $\mu_{i}=m_{i} / m_{C}$. The coordinates in the body fixed frame is $\delta_{1}=\left(x_{1 m}, y_{1 m}, z_{1 m}\right)^{T}$, $i=1,2, \ldots, n$. In the ground frame, the velocity of the center of MMAV is $V_{A B}=\dot{\delta}_{A B}$, and the acceleration is $\dot{V}_{A B}=\ddot{\delta}_{A B}$. Let the coordinates in the ground with the body fixed frame of the $i$ th moving mass be $\delta_{d i}$, let the coordinates in the ground frame be $\delta_{A i}$. There are the relationships $\delta_{d i}=L_{A 1} \delta_{1 i}, \delta_{A i}=$ $\delta_{d i}+\delta_{A B}$.

The coordinates of MMAV's c. $\mathrm{m}$. in the ground frame are given by

$$
\begin{aligned}
\delta_{A C} & =\frac{1}{m_{C}}\left(m_{B} \delta_{A B}+\sum_{i=1}^{n} m_{i} \delta_{A i}\right) \\
& =\delta_{A B}+\sum_{i=1}^{n} \mu_{i} L_{A 1} \delta_{1 i} \\
& =\delta_{A B}+L_{A 1} \sum_{i=1}^{n} \mu_{i} \delta_{1 i} .
\end{aligned}
$$

After derivation, the translational equation of the MMAV in the ground frame can be presented as follows:

$$
\begin{aligned}
\dot{V}_{A B}= & \frac{R}{m_{C}}+g-\left(\left(\omega_{1}\right)_{A}^{\times^{*}}+\left(\omega_{1}\right)_{A}^{\times^{2}}\right) L_{A 1} \sum_{i=1}^{n} \mu_{i} \delta_{1 i} \\
& -2\left(\omega_{1}\right)_{A}^{\times} L_{A 1} \sum_{i=1}^{n} \mu_{i} \dot{\delta}_{1 i}-L_{A 1} \sum_{i=1}^{n} \mu_{i} \ddot{\delta}_{1 i} .
\end{aligned}
$$

Then, the translational equation of the MMAV in the body fixed frame is as follows:

$$
\begin{aligned}
\dot{V}_{1 B}+\left(\omega_{1}\right)_{1}^{\times} V_{1 B}= & \frac{R_{1}}{m_{C}}+g_{1}-\left(\left(\omega_{1}\right)_{1}^{\times^{*}}+\left(\omega_{1}\right)_{1}^{\times^{2}}\right) \sum_{i=1}^{n} \mu_{i} \delta_{1 i} \\
& -2\left(\omega_{1}\right)_{1}^{\times} \sum_{i=1}^{n} \mu_{i} \dot{\delta}_{1 i}-\sum_{i=1}^{n} \mu_{i} \ddot{\delta}_{1 i},
\end{aligned}
$$

where $L_{A 1}$ is the transformation matrix from the body fixed frame to the ground frame, $\left(\omega_{1}\right)_{A}^{\times}$is the antisymmetry matrix of the angular velocity of the MMAV in the ground frame. $\left(\omega_{1}\right)_{1}^{\times}$is the antisymmetry matrix of the angular velocity of the MMAV in the body fixed frame.

Correspondingly, the force equation of the $i$ th moving mass in the body fixed frame is given by

$$
\begin{aligned}
F_{1 i}= & \mu_{i} R_{1} \\
& +m_{i}\left[\left(\left(\omega_{1}\right)_{1}^{\times^{*}}+\left(\omega_{1}\right)_{1}^{\times^{2}}\right) \delta_{1 i}+2\left(\omega_{1}\right)_{1}^{\times} \dot{\delta}_{1 i}+\ddot{\delta}_{1 i}\right] \\
& -m_{i}\left[\left(\left(\omega_{1}\right)_{1}^{\times^{*}}+\left(\omega_{1}\right)_{1}^{\times^{2}}\right) \sum_{i=1}^{n} \mu_{i} \delta_{1 i}\right. \\
& \left.+2\left(\omega_{1}\right)_{1}^{\times} \sum_{i=1}^{n} \mu_{i} \dot{\delta}_{1 i}+\sum_{i=1}^{n} \mu_{i} \ddot{\delta}_{1 i}\right] .
\end{aligned}
$$




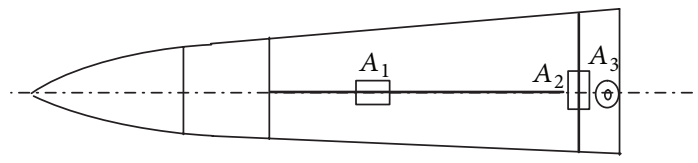

FIgURE 1: MMAV with three moving masses.

According to D'Alembert principle, the rotational equation in body coordinates is obtained as follows:

$$
\left(\frac{d H}{d t}\right)_{1}+M_{1 F}=\frac{d H_{1}}{d t}+\left(\omega_{1}\right)_{1}^{\times} H_{1}+M_{1 F}=M_{1 R}
$$

where, $M_{1 F}=-\sum_{i=1}^{n} S_{1 i} F_{1 i}, S_{1 i}$ is the antisymmetric matrix of the $i$ th moving mass in the body fixed frame representing the position coordinates $\delta_{1 i}$.

2.2. The Dynamical Model of the MMAV with Three Moving Masses. The structure diagram of the MMAV with three moving masses is shown in Figure 1. To quickly adjust the flying attitude and decrease coupling, one mass is fixed at the $x$-axis in the body fixed frame. Another two masses are fixed at the $y$-axis and the $z$-axis in radial direction through the MMAV's axis.

The mass of the MMAV's shell is $m_{B}$. The mass of axial moving mass $A_{1}$ is $m_{1}$, and the coordinate in the body fixed frame is $\delta_{11}$. The mass of radial moving mass $A_{2}$ is $m_{2}$, and the coordinate in the body fixed frame is $\delta_{12}$. The mass of radial moving mass $A_{3}$ is $m_{3}$ and the coordinate in the body fixed frame is $\delta_{13}$. So, the total mass is $m_{C}=m_{B}+m_{1}+m_{2}+m_{3}$, and the mass ratios are $\mu_{1}=m_{1} / m_{C}, \mu_{2}=m_{2} / m_{C}$, and $\mu_{3}=$ $m_{3} / m_{C}$, respectively.

This section derives the equations of motion fully accounting for the dynamic coupling between the four bodies. The moving masses are allowed to translate with respect to the MMAV's shell but are not allowed to rotate with respect to the MMAV's shell. Both the MMAV and the moving masses are assumed to be rigid bodies.

In the body fixed frame, the interaction between axial moving mass $A_{1}$ and MMAV is $F_{11}$, the interaction between radial moving mass $A_{2}$ and MMAV is $F_{12}$, and the interaction between radial moving mass $A_{3}$ and MMAV is $F_{13}$. Equation (4) can be presented below

$$
\begin{aligned}
& F_{11}=m_{1} {\left[\dot{V}_{1 B}+\left(\omega_{1}\right)_{1}^{\times} V_{1 B}+\left(\left(\omega_{1}\right)_{1}^{x^{*}}+\left(\omega_{1}\right)_{1}^{x^{2}}\right) \delta_{11}\right.} \\
&\left.+2\left(\omega_{1}\right)_{1}^{\times} \dot{\delta}_{11}+\ddot{\delta}_{11}-g_{1}\right], \\
& F_{12}=m_{1} {\left[\dot{V}_{1 B}+\left(\omega_{1}\right)_{1}^{\times} V_{1 B}+\left(\left(\omega_{1}\right)_{1}^{x^{*}}+\left(\omega_{1}\right)_{1}^{x^{2}}\right) \delta_{12}\right.} \\
&+\left.2\left(\omega_{1}\right)_{1}^{\times} \dot{\delta}_{12}+\ddot{\delta}_{12}-g_{1}\right], \\
& F_{13}=m_{3}\left[\dot{V}_{1 B}+\left(\omega_{1}\right)_{1}^{\times} V_{1 B}+\left(\left(\omega_{1}\right)_{1}^{x^{*}}+\left(\omega_{1}\right)_{1}^{x^{2}}\right) \delta_{13}\right. \\
&\left.+2\left(\omega_{1}\right)_{1}^{\times} \dot{\delta}_{13}+\ddot{\delta}_{13}-g_{1}\right] .
\end{aligned}
$$

The vector translational dynamics of MMAV can be obtained by (3) as follows

$$
\begin{aligned}
& \dot{V}_{1 B}+\left(\omega_{1}\right)_{1}^{\times} V_{1 B}+\left(\omega_{1}\right)_{1}^{\times^{*}}\left(\mu_{1} \delta_{11}+\mu_{2} \delta_{12}+\mu_{3} \delta_{13}\right) \\
&=\frac{R_{1}}{m_{C}}+g_{1}-[\left(\omega_{1}\right)_{1}^{\times^{2}}\left(\mu_{1} \delta_{11}+\mu_{2} \delta_{12}+\mu_{3} \delta_{13}\right) \\
&+ 2\left(\omega_{1}\right)_{1}^{\times}\left(\mu_{1} \dot{\delta}_{11}+\mu_{2} \dot{\delta}_{12}+\mu_{3} \dot{\delta}_{13}\right) \\
&\left.+\left(\mu_{1} \ddot{\delta}_{11}+\mu_{2} \ddot{\delta}_{12}+\mu_{3} \ddot{\delta}_{13}\right)\right] .
\end{aligned}
$$

The rotational dynamics of MMAV obtained by (5) are given by (8):

$$
\begin{aligned}
& {\left[J_{1}+\left(1-\mu_{1}\right) m_{1} S_{11}^{T} S_{11}+\left(1-\mu_{2}\right) m_{2} S_{12}^{T} S_{12}\right.} \\
& +\left(1-\mu_{3}\right) m_{3} S_{13}^{T} S_{13}-\mu_{2} m_{1} S_{11} S_{12}^{T}-\mu_{3} m_{1} S_{11} S_{13}^{T} \\
& -\mu_{1} m_{2} S_{12} S_{11}^{T}-\mu_{3} m_{2} S_{12} S_{13}^{T}-\mu_{1} m_{3} S_{13} S_{11}^{T} \\
& \left.-\mu_{2} m_{3} S_{13} S_{12}^{T}\right]\left(\omega_{1}\right)_{1}^{*} \\
& =M_{1 R}-\left(\mu_{1} S_{11}+\mu_{2} S_{12}+\mu_{3} S_{13}\right) R_{1}-\left(\omega_{1}\right)_{1}^{\times} J_{1}\left(\omega_{1}\right)_{1} \\
& \quad+\left[\mu_{1} m_{2} S_{12}+\mu_{1} m_{3} S_{13}-\left(1-\mu_{1}\right) m_{1} S_{11}\right] \\
& \quad \times\left(\left(\omega_{1}\right)_{1}^{\times 2} \delta_{11}+2\left(\omega_{1}\right)_{1}^{\times} \dot{\delta}_{11}+\ddot{\delta}_{11}\right) \\
& \quad+\left[\mu_{2} m_{1} S_{11}+\mu_{2} m_{3} S_{13}-\left(1-\mu_{2}\right) m_{2} S_{12}\right] \\
& \quad \times\left(\left(\omega_{1}\right)_{1}^{\times 2} \delta_{12}+2\left(\omega_{1}\right)_{1}^{\times} \dot{\delta}_{12}+\ddot{\delta}_{12}\right) \\
& \quad+\left[\mu_{3} m_{1} S_{11}+\mu_{3} m_{2} S_{12}-\left(1-\mu_{3}\right) m_{3} S_{13}\right] \\
& \quad \times\left(\left(\omega_{1}\right)_{1}^{\times 2} \delta_{13}+2\left(\omega_{1}\right)_{1}^{\times} \dot{\delta}_{13}+\ddot{\delta}_{13}\right),
\end{aligned}
$$

where $J_{1}$ is the $3 \times 3$ inertia tensor of MMAV about its center of mass; the antisymmetric matrix of position coordinates of the three moving masses in the body fixed frame are $S_{11}, S_{12}$, and $S_{13}$, respectively.

Furthermore, the equations of motion of MMAV system also include some relative movement functions and nonlinear aerodynamic functions. The equations of motion clearly indicate that the MMCS is a complex nonlinear system which has the variable coefficients and large disturbances caused by the accelerations and velocities of masses.

\section{Sliding Mode Control System Design}

3.1. Hierarchy-Structured Dynamic Inversion of MMAV. Although the design of MMCS using moving masses actuation appears to be conceptually straightforward, difficulties arise as a result of the highly coupled and nonlinear nature of the system dynamics. A major concern is how to control moving mass position coordinately to satisfy the needed moment. 
Dynamic inversion is one of the nonlinear flight control techniques based on feedback linearization. Consider the following MMAV nonlinear system [32]:

$$
\begin{gathered}
\dot{x}=f(x)+g(x) u, \\
y=h(x),
\end{gathered}
$$

where $x=\left[\begin{array}{llllllll}\omega_{x 1} & \omega_{y 1} & \omega_{z 1} & \vartheta & \psi & \gamma & n_{y} & n_{z}\end{array}\right]^{T} \in R^{8}$ is the state variable, $u=\left[\begin{array}{lll}\delta_{x} & \delta_{y} & \delta_{z}\end{array}\right]^{T} \in R^{3}$ is the control input, and $y$ is the output to be controlled by the control input $u$. Differentiating $y$ in (10), in general, one obtains

$$
\dot{y}=\frac{\partial h}{\partial x}(f(x)+g(x) u)=F(x, u) .
$$

It is assumed here that $F(x, u)$ is invertible with respect to $u$. Then, consider the following nonlinear feedback control using the inverse dynamics of (11):

$$
u=F^{-1}(x, v)
$$

where $v$ is the auxiliary input. Substituting (12) to (11), one obtains

$$
\dot{y}=F\left(x, F^{-1}(x, v)\right)=v .
$$

Equation (13) shows that the relation between the output and the auxiliary input is now feedback linearized. $v$ is often given as shown in (14), so that (13) will form a first-order servo system,

$$
v=K\left(y_{c}-y\right)
$$

where $K \in R^{3}$ is a feedback gain matrix and $y_{c}$ is a commanded value for $y$. Feedback linearization term in $u$ cancels out the inherent stability of the dynamical system, and then the outer tracking loop given in (14) rearranges the corresponding nonlinear modes to realize the desired output dynamics $y_{c}$.

But it is known that the number of the modes that dynamic inversion can rearrange is equal to the sum of the relative degrees of the input-to-output relations and that the remaining modes are unobservable from $y$. These unobservable modes are called zero dynamics. If some of the input-tooutput relations are nonminimum phase, the desired output dynamics $y_{c}$ cannot be realized for the reason that the corresponding zeros are located in the right half plane and, therefore, result in unstable zero dynamics. Unfortunately, some input-to-output relations in the aircraft dynamics are nonminimum phase due to the derivatives of aerodynamic forces with respect to the control surface deflections. This fact prevents direct application of dynamic inversion to MMCS.

The problem can be avoided by two time-scale separation, where the dynamics are separated into the fast one and the slow one according to the time-scales of the variables. The fast variables are used to control the slow state variables, and the fast variables are controlled by the control input. The MMAV nonlinear system is rewritten as follows:

$$
\begin{gathered}
\dot{x}_{f}=f_{f 1} x_{f}+f_{s 1} x_{s}+g_{f} u, \\
\dot{x}_{s}=f_{f 2} x_{f}+f_{s 2} x_{s}+g_{s} u, \\
y=h\left(x_{s}\right),
\end{gathered}
$$

where $x_{f}=\left[\begin{array}{lll}\omega_{x 1} & \omega_{y 1} & \omega_{z 1}\end{array}\right]^{T} \in R^{3}$ is the fast state, $x_{s}=$ $\left[\begin{array}{lllll}\vartheta & \psi & \gamma & n_{y} & n_{z}\end{array}\right] \in R^{5}$ is the slow state, $u=\left[\begin{array}{lll}\delta_{x} & \delta_{y} & \delta_{z}\end{array}\right]^{T} \in$ $R^{3}$ is the control input, and $y$ is the output to be controlled. For simplicity, the output to be controlled is assumed to be $y=x_{s}$. Then, the input-to-output relations in slow time-scale can be derived as follows:

$$
\dot{y}=f_{f 2} x_{f}+f_{s 2} x_{s}+g_{s} u
$$

One obtains $x_{f c}$ from (18), which is the commanded values for $x_{f}$, using dynamic inversion as follows:

$$
\begin{gathered}
x_{f c}=f_{f 2}^{-1}\left(v_{s}-f_{s 2} x_{s}\right)-f_{f 2}^{-1} g_{s} u, \\
v_{s}=K_{s}\left(y_{c}-y\right),
\end{gathered}
$$

where $v_{s}$ is the auxiliary input for slow time-scale controller and $K_{s} \in R^{5}$ is the feedback gain matrix. If $x_{f}=x_{f c}$, and the following relation holds

$$
\dot{y}=v_{s}=K_{s}\left(y_{c}-y\right) .
$$

Finally, $u_{c}$ is derived in fast time-scale as follows using dynamic inversion again so that $x_{f}$ will follow its command $x_{f c}$. Consider

$$
\begin{gathered}
u_{c}=g_{f}^{-1}\left(v_{f}-f_{f 1} x_{f}-f_{s 1} x_{s}\right), \\
v_{f}=K_{f}\left(x_{f c}-x_{f}\right),
\end{gathered}
$$

where $v_{f}$ is the auxiliary input for fast time-scale controller and $K_{f} \in R^{3}$ is the feedback gain matrix.

Note that if $x_{f}=x_{f c}$ then the asymptotic stability of $y$ in (20) is not necessarily guaranteed but depends on the fast dynamics. From the viewpoint of the singular perturbation theory, the asymptotic stability is guaranteed, when the two dynamics are well separated; that is, the time-scales are not very close to each other. Although no theoretical background of the asymptotic stability of the slow variable is given in this paper, it will be evaluated by linearization and 6DOF nonlinear simulation. Two time-scale separation is easily expanded into multi-time-scale separation. Dynamic inversion using multi-time-scale separation and multiloop closure method is called hierarchy-structured dynamic inversion [33]. The following section details chattering free fuzzy sliding mode control using dynamic inversion to MMAV systems.

3.2. Chattering Free Fuzzy Sliding Mode Control of MMAV. Without loss of generality, the parameter uncertainty and external disturbance are taken into account in the MMAV 
control system. Then, the dynamics equations (15) and (16) can be rewritten in abbreviate form as:

$$
\begin{gathered}
\dot{x}_{f}=f_{f 1} x_{f}+f_{s 1} x_{s}+\Delta f_{f}+g_{f} u+d_{f}, \\
\dot{x}_{s}=f_{f 2} x_{f}+f_{s 2} x_{s}+\Delta f_{s}+g_{s} u+d_{s},
\end{gathered}
$$

where $\Delta f_{f}$ and $\Delta f_{s}$ denote uncertain terms representing the unmodeled dynamics or structural variation of the MMAV system, which is owing to the time variations of the atmospheric coefficients system, $d_{f}$ and $d_{s}$ denote the disturbance of system.

In the practical MMAV system, the uncertain term $\Delta f_{f}$, $\Delta f_{s}$ and the disturbance term $d_{f}, d_{s}$ are bounded, that is, $\left\|\Delta f_{f}\right\|_{\infty} \leq \alpha_{f},\left\|\Delta f_{s}\right\|_{\infty} \leq \alpha_{s}$ and $\left\|d_{f}\right\|_{\infty} \leq \beta_{f},\left\|d_{s}\right\|_{\infty} \leq \beta_{s}$, where $\alpha_{f}, \alpha_{s}, \beta_{f}, \beta_{s}$ are four positive and known constants.

The control problem of a practical system is to get the system to track an $n$-dimensional desired vector $x_{d}(t), x_{d}(t)=$ $\left[\begin{array}{llll}x_{d 1}(t) & x_{d 2}(t) & \cdots & x_{d n}(t)\end{array}\right]^{T} \in R^{n}$, which belongs to a class of continuous functions on $\left[t_{0}, \infty\right]$. Let the tracking error be

$$
e(t)=x_{d}(t)-x(t)=\left[\begin{array}{llll}
e_{1}(t) & e_{2}(t) & \cdots & e_{n}(t)
\end{array}\right]^{T} .
$$

The control goal is that, for any given target $x_{d}(t)$, a sliding mode control (SMC) is designed, such that the resulting state response of the tracking error vector satisfies

$$
\lim _{t \rightarrow \infty}\|e(t)\|=\lim _{t \rightarrow \infty}\left\|x_{d}(t)-x(t)\right\| \longrightarrow 0,
$$

where $\|\cdot\|$ denotes the Euclidean norm of a vector.

$\mathrm{SMC}$ is an efficient tool to control complex high-order dynamic plants operating under uncertainty conditions due to its order reduction property and low sensitivity to disturbances and plant parameter variations. In SMC, the states of the controlled system are first guided to reside on a designed surface (i.e., the sliding surface) in state space and then confined there with a shifting law (based on the system states). A time varying surface $s(t)$ is defined in the state space $R^{n}$ by equating the variable $s(x)$, defined below, to zero,

$$
s(x)=\left(\frac{d}{d t}+\lambda\right)^{n-1} e(t) .
$$

Here, $\lambda$ is a strict positive constant, taken to be the bandwidth of the system [17]. As our problem formulation is a firstorder differential equation, then $n=1$, and the relation (26) can be rewritten as follows:

$$
s=e(t)
$$

When the closed loop system is in the sliding mode, it satisfies $\dot{s}_{f}=0$, and then the equivalent control law of the fast dynamics of the MMAV system is obtained by

$$
u_{\mathrm{eq}}=g_{f}^{-1}\left(\dot{x}_{f d}-f_{f 1} x_{f}-f_{s 1} x_{s}-\Delta f_{f}-d_{f}\right) .
$$

In practical systems, the system uncertainty $\Delta f_{f}$ and external disturbance are unknown, and the implemented equivalent control input is modified as

$$
u_{\mathrm{eq}}=g_{f}^{-1}\left(\dot{x}_{f d}-f_{f 1} x_{f}-f_{s 1} x_{s}\right) .
$$

According to the Lyapunov stability theory [17], a Lyapunov function is defined as

$$
V_{f}=\frac{1}{2} s_{f}^{T} s_{f}
$$

Then, the derivative of $V_{f}$ becomes

$$
\dot{V}_{f}=s_{f}^{T} \dot{s}_{f}=s_{f}^{T}\left(\dot{x}_{f d}-\dot{x}_{f}\right) .
$$

In the above equation, if $\dot{V}_{f}$ is negative for all $s_{f} \neq 0$, then the so-called reaching condition [17] is satisfied. That is, the control $u_{f}$ is designed to guarantee that the states are hitting on the sliding surface $s_{f}=0$.

The reaching control law is selected as $u_{r}=g_{f}^{-1} k_{w f} \operatorname{sgn}\left(s_{f}\right)$, and the overall control $u$ is determined by

$$
u=u_{\mathrm{eq}}+u_{r}=u_{\mathrm{eq}}+g_{f}^{-1} k_{w f} \operatorname{sgn}\left(s_{f}\right)
$$

where $k_{w f}$ is the switching gain.

Based on the Lyapunov theory, the fast dynamics states approach the hyperplane, if $\dot{V}_{f} \leq-k_{w f}\left|s_{f}\right|$. The error vector asymptotically reduces to zero once the system states are on $s_{f}=0$.

The finite time delays and limitations of practical control systems render the implementation of such control signals problematic in real-world systems. In other words, the sign function in overall control $u$ will cause the control input to produce the chattering phenomenon. In the current study, this problem is resolved through the application of a fuzzy logic control (FLC) scheme to determine an appropriate reaching law. Furthermore, if system uncertainties are large, the sliding mode controller would require a high switching gain with a thicker boundary layer to eliminate the resulting higher chattering effect. However, if we continuously increase the boundary layer thickness, we are actually reducing the feedback system to a system without a sliding mode. To tackle these difficulties, recently, FSMC has also been used for this purpose, which is shown to be quite effective [34, 35].

In this paper, in order to eliminate the chattering problem, a fuzzy inference engine is used for reaching phase, and fuzzy sliding mode control methodology is proposed. The main advantage of this method is that the robust behavior of the system is guaranteed. The second advantage of the proposed scheme is that the performance of the system in the sense of removing chattering is improved in comparison with the same SMC technique without using FLC.

The equivalent control part is the same as that in (29), and the reaching law is selected as

$$
u_{r}=g_{f}^{-1} k_{f s} u_{f s}
$$

where $k_{f s}$ is the normalization factor of the output variable, and $u_{f s}$ is the output of the FSMC, which is determined by the normalized $s_{f}$ and $\dot{s}_{f}$.

The fuzzy control rules can be represented as the mapping of the input linguistic variables $s_{f}$ and $\dot{s}_{f}$ to the output linguistic variable $u_{f s}$ as follows:

$$
u_{f s}=\operatorname{FSMC}\left(s_{f}, \dot{s}_{f}\right)=\left[\begin{array}{lll}
u_{f s(1)} & u_{f s(2)} & u_{f s(3)}
\end{array}\right]^{T} .
$$




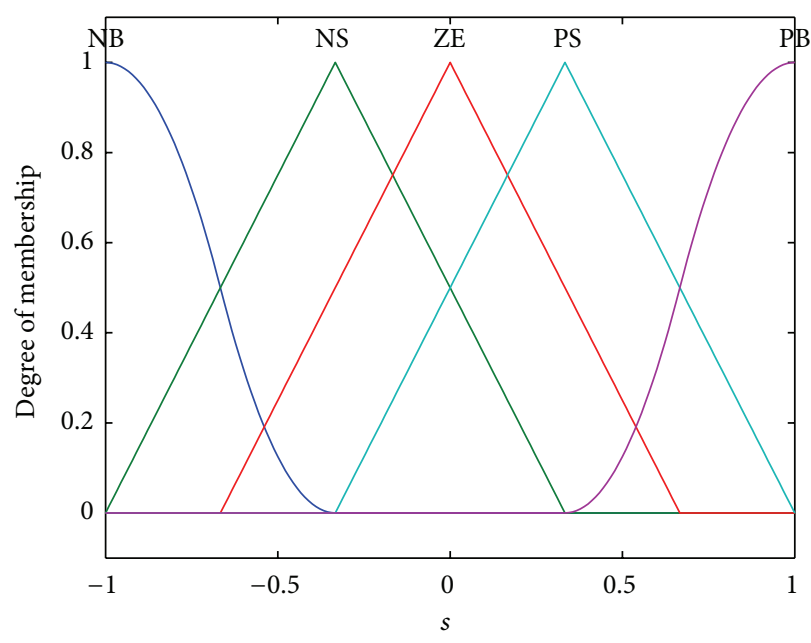

(a)

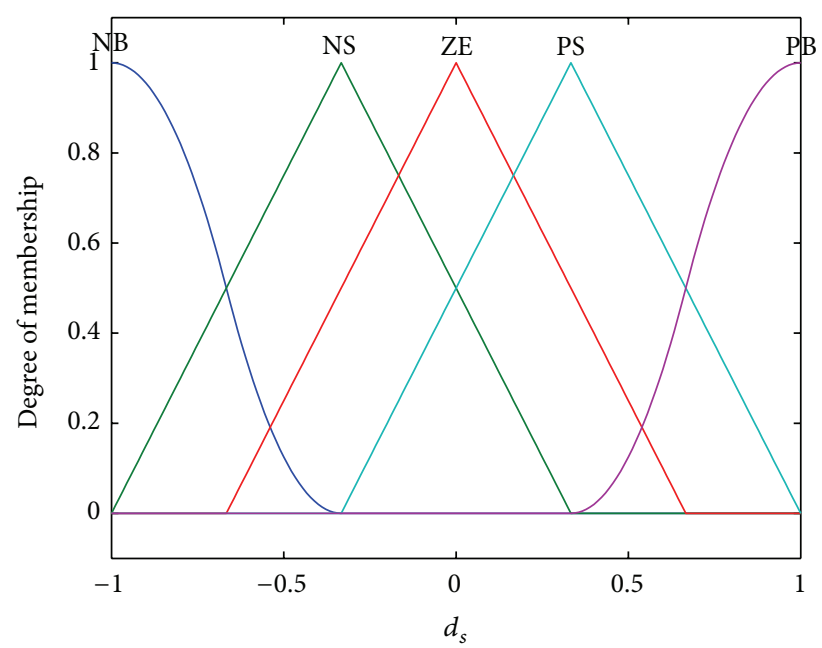

(b)

FIGURE 2: Membership function of $s_{f}, \dot{s}_{f}$ and $s_{s}, \dot{s}_{s}$.

The membership function of input linguistic for each set of variables $s_{f i}$ and $\dot{s}_{f i}$ and the membership functions of the output linguistic variable $u_{f_{s}(i)}, i=1,2,3$, are shown in Figure 2 , respectively. Here, $u_{f s(i)}$ is denoted as

$$
u_{f s(i)}=\operatorname{FSMC}\left(s_{f i}, \dot{s}_{f i}\right) .
$$

Our proposed FLC has two inputs and one output. These are $s_{f}, \dot{s}_{f}$, and the control signal, respectively. Linguistic variables which imply inputs and outputs have been classified as: NB, NM, NS, ZE, PS, PM, and PB. Inputs and outputs are all normalized in the interval of $[-1,1]$ with equal span as shown in Figure 2. The linguistic labels used to describe the fuzzy sets are "Negative Big" (NB), "Negative Small" (NS), "Zero" (ZE), "Positive Small" (PS), and "Positive Big" (PB). It is possible to assign a set of decision rules as shown in Table 1. The fuzzy rules are extracted in such a way that the stability of the system would be satisfied, which was explained in more detail before. These rules contain the input/output relationships that define the control strategy. Each control input has seven fuzzy sets so that there are at most 25 fuzzy rules. Consider

$$
u=u_{\mathrm{eq}}+g_{f}^{-1} k_{f s} u_{f s}=g_{f}^{-1}\left(\dot{x}_{f d}-f_{f 1} x_{f}-f_{s 1} x_{s}+k_{f s} u_{f s}\right) .
$$

In the following theorem, proposed scheme (36) will be proved to be able to drive the nonlinear system (22) onto the sliding surface $s(x)=0$. That is, the reaching condition $s(x) \dot{s}(x)<0$ is guaranteed.

Theorem 1. Consider the uncertain nonlinear system (22) controlled by $u$ in (36), where $u_{e q}$ is in (29), $u_{f s}$ is in (34) and $k_{f s}>\alpha_{f}+\beta_{f}$. Then, the error state trajectory converges to the sliding surface $s_{f}(x)=0$.
TABLE 1: Rule-table of FSMC.

\begin{tabular}{llccccc}
\hline$u_{f s}, u_{s s}$ & & \multicolumn{5}{c}{$s_{f}, s_{s}$} \\
& & NB & NS & ZO & PS & PB \\
\hline \multirow{4}{*}{$\dot{s}_{f}, \dot{s}_{s}$} & NB & PB & PB & PS & PS & ZO \\
& NS & PB & PS & PS & ZO & ZO \\
& ZO & PS & PS & ZO & ZO & NS \\
& PS & PS & ZO & ZO & NS & NS \\
& PB & ZO & ZO & NS & NS & NB \\
\hline
\end{tabular}

Proof. Let $V_{f}=(1 / 2) s_{f}^{T} s_{f}$, then

$\dot{V}_{f}$

$$
\begin{aligned}
& =s_{f}^{T} \dot{s}_{f}=s_{f}^{T} \cdot\left(\dot{x}_{f d}-\dot{x}_{f}\right) \\
& =s_{f}^{T} \cdot\left(\dot{x}_{f d}-f_{f 1} x_{f}-f_{s 1} x_{s}-\Delta f_{f}-g_{f} u-d_{f}\right) \\
& =s_{f}^{T} \cdot\left(-k_{f s} u_{f s}-\Delta f_{f}-d_{f}\right) \\
& =s_{f}^{T} \cdot\left[-k_{f s}\right. \\
& \quad \times\left(\operatorname{FSMC}\left(s_{f 1}, \dot{s}_{f 1}\right) \operatorname{FSMC}\left(s_{f 2}, \dot{s}_{f 2}\right) \operatorname{FSMC}\left(s_{f 3}, \dot{s}_{f 3}\right)\right)^{T} \\
& \left.\quad-\Delta f_{f}-d_{f}\right] \\
& \leq k_{f s}\left[s_{f 1} \times \operatorname{FSMC}\left(s_{f 1}, \dot{s}_{f 1}\right)+s_{f 2} \times \operatorname{FSMC}\left(s_{f 2}, \dot{s}_{f 2}\right)\right. \\
& \left.\quad+s_{f 3} \times \operatorname{FSMC}\left(s_{f 3}, \dot{s}_{f 3}\right)\right]+\alpha_{f}\left\|s_{f}\right\|+\beta_{f}\left\|s_{f}\right\| \\
& \leq k_{f s}\left[-\left|s_{f 1}\right|-\left|s_{f 2}\right|-\left|s_{f 3}\right|\right]+\alpha_{f}\left\|s_{f}\right\|+\beta_{f}\left\|s_{f}\right\| \\
& =-k_{f s}\left[\left|s_{f 1}\right|+\left|s_{f 2}\right|+\left|s_{f 3}\right|\right]+\alpha_{f}\left\|s_{f}\right\|+\beta_{f}\left\|s_{f}\right\|
\end{aligned}
$$




$$
\begin{aligned}
& \leq \alpha_{f}\left\|s_{f}\right\|+\beta_{f}\left\|s_{f}\right\|-k_{f s}\left\|s_{f}\right\| \\
& =-\left[k_{f s}-\left(\alpha_{f}+\beta_{f}\right)\right]\left\|s_{f}\right\| .
\end{aligned}
$$

So, if we select $k_{f s}>\alpha_{f}+\beta_{f}$, one can conclude that the reaching condition $s_{f} \dot{s}_{f}<0$ is always satisfied. Thus, the proof is achieved completely.

Theorem 2. One obtains $x_{f c}$ from (23), which is the commanded values for $x_{f}$, using dynamic inversion as follows:

$$
x_{f c}=f_{f 2}^{-1}\left(\dot{x}_{s d}-f_{s 2} x_{s}-k_{s s} u_{s s}\right)-f_{f 2}^{-1} g_{s} u .
$$

If $k_{s s}>\alpha_{s}+\beta_{s}$ is satisfied, then the error state trajectory converges to the sliding surface $s_{f}(x)=0$.

Proof. According to the Lyapunov stability theory [17], a Lyapunov function of the slow dynamics of the MMAV system is defined as

$$
V_{s}=\frac{1}{2} s_{s}^{T} s_{s}
$$

Then, the derivative of $V_{s}$ becomes

$$
\begin{aligned}
& \dot{V}_{s}=s_{s}^{T} \dot{s}_{s}=s_{s}^{T}\left(\dot{x}_{s d}-\dot{x}_{s}\right), \\
& \dot{V}_{s}= s_{s}^{T} \cdot \dot{s}_{s} \\
&= s_{s}^{T} \cdot\left(\dot{x}_{s d}-f_{f 2} x_{f}-f_{s 2} x_{s}-\Delta f_{s}-g_{s} u-d_{s}\right) \\
&= s_{s}^{T} \cdot\left(-k_{s s} u_{s s}-\Delta f-d_{s}\right) \\
&= s_{s}^{T} \cdot\left[-k_{s s}\left(\mathrm{FSMC}\left(s_{s 1}, \dot{s}_{s 1}\right) \quad \ldots \quad \operatorname{FSMC}\left(s_{s 5}, \dot{s}_{s 5}\right)\right)^{T}\right. \\
&\left.\quad-\Delta f_{s}-d_{s}\right] \\
& \leq k_{s s}\left[s_{s 1} \times \mathrm{FSMC}\left(s_{s 1}, \dot{s}_{s 1}\right)+\cdots+s_{s 5} \times \mathrm{FSMC}\left(s_{s 5}, \dot{s}_{s 5}\right)\right] \\
&+\alpha_{s}\left\|s_{s}\right\|+\beta_{s}\left\|s_{s}\right\| \\
& \leq k_{s s}\left[-\left|s_{s 1}\right|-\left|s_{s 2}\right|-\left|s_{s 3}\right|-\left|s_{s 4}\right|-\left|s_{s 5}\right|\right] \\
&+\alpha_{s}\left\|s_{s}\right\|+\beta_{s}\left\|s_{s}\right\| \\
&=-k_{s s}\left[\left|s_{s 1}\right|+\left|s_{s 2}\right|+\left|s_{s 3}\right|+\left|s_{s 4}\right|+\left|s_{s 5}\right|\right] \\
&+\alpha_{s}\left\|s_{s}\right\|+\beta_{s}\left\|s_{s}\right\| \\
& \leq \alpha_{s}\left\|s_{s}\right\|+\beta_{s}\left\|s_{s}\right\|-k_{s s}\left\|s_{s}\right\| \\
&=-\left[k_{s s}-\left(\alpha_{s}+\beta_{s}\right)\right]\left\|s_{s}\right\| .
\end{aligned}
$$

So, if we select $k_{s s}>\alpha_{s}+\beta_{s}$, one can conclude that the reaching condition $s_{s} \dot{s}_{s}<0$ is always satisfied. This completes the proof.

\section{Simulation Results and Discussions}

In order to demonstrate the performance of the proposed flight control system, simulations are presented in this section. The initial conditions of the engagement are given in [25]. The transfer function of MMAV's actuator masses is described as follows:

$$
G_{R}(s)=\frac{1}{T_{R}^{2} s^{2}+2 \zeta_{R} T_{R} s+1},
$$

where $T_{R}$ is the equivalent time constant, and $\zeta_{R}$ is damping ratio of three moving mass. The time constant and damping ratio are tuned precisely in order to obtain the best possible performances in response to a command signal. The time constant and the damping ratio of axial moving mass are chosen as 0.01 and 0.8 , and the time constant and the damping ratio of radial moving mass are chosen as 0.005 and 0.8 , respectively.

The needed moment coefficients and aerodynamic coefficients are also determined through a looked-up table using the current flight status (i.e., Mach number, altitude). Moreover, without loss of generality, in all the simulations, the uncertainty/disturbance terms in the dynamics equations are randomly selected within $15 \%$ of their nominal values in all the simulations.

Furthermore, in order to show the full potential of the proposed control system, the controller parameters (i.e., $k_{f}$, $k_{s s}$ ) are optimally chosen using a genetic algorithm (GA) [36]. In which case, the cost function subjected to be minimized is

$$
J=\sum u^{T} W_{u} u+s_{f}^{T} W_{f} s_{f}+s_{s}^{T} W_{s} s_{s},
$$

where $W_{u}, W_{f}$, and $W_{s}$ are the weighting matrices.

Genetic algorithms (GA) search the solution space of a function through the use of simulated evolution, that is, the survival of the first strategy. In general, the fittest individuals of any population tend to reproduce and survive to the next generation, thus, improving successive generations. However, inferior individuals can, by chance, survive and also reproduce. GA have been shown to solve linear and nonlinear problems by exploring all regions of the state space and exponentially exploiting promising areas through mutation, crossover, and selection operations applied to individuals in the population. Thus, the main advantage of using GA is that they do not get trapped in local minimal, and they can use any cost function that can be computed in a reasonable amount of time.

In practical applications, the acceleration commands $\left(a_{c}=g n_{c}\right)$ are determined by a guidance loop. According to the magnitude and frequency of spiral maneuvering targets [37], the command $a_{y c}$ and $a_{z c}$ are given by sinusoidal signals with amplitude 10 and frequency $3 \mathrm{rad} / \mathrm{s}$.

The mathematical simulation of MMAV is performed using sliding mode control and fuzzy sliding mode control based on dynamic inversion designed in this paper. The simulation results are shown in Figures 3 and 4, respectively.

In Figures 3(d), 3(e), 4(d), and 4(e), the dotted line, are given acceleration commands, and the solid line, are actual acceleration commands. The results in the figures show 


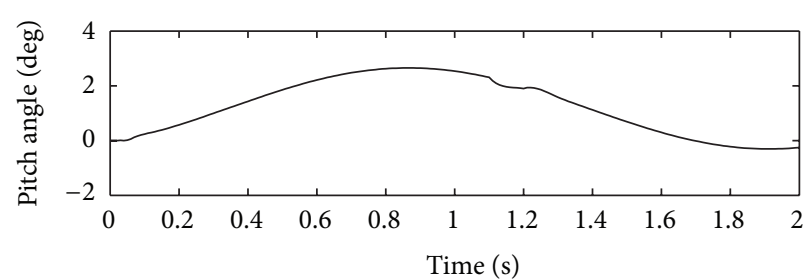

(a)

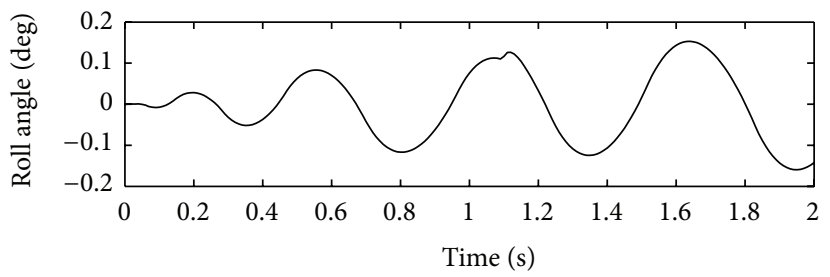

(c)

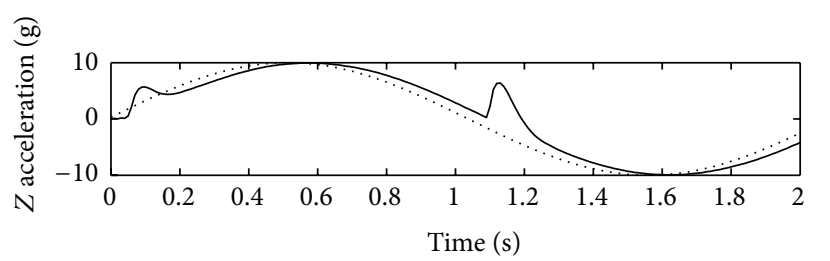

$\begin{array}{ll}\ldots \ldots & a_{z c} \\ - & a_{z}\end{array}$

(e)

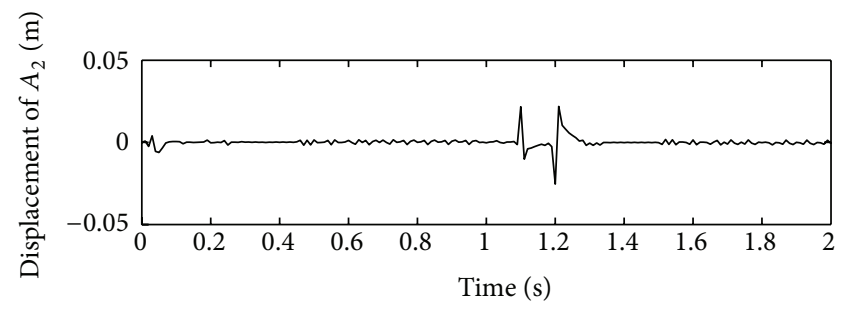

(g)

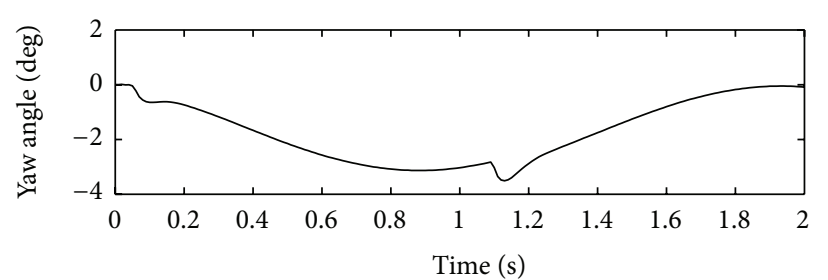

(b)

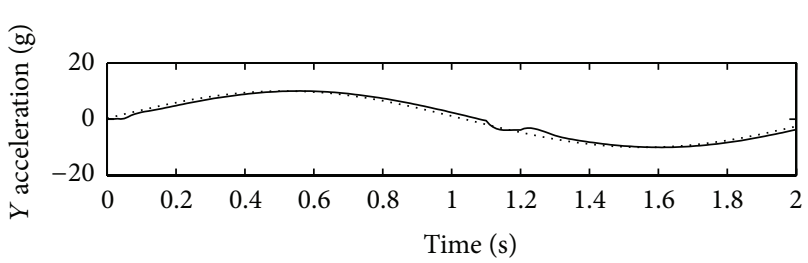

$\ldots+a_{y c}$

$-a_{y}$

(d)

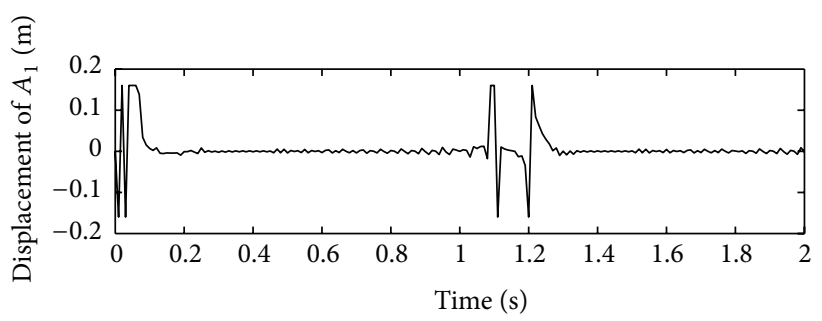

(f)

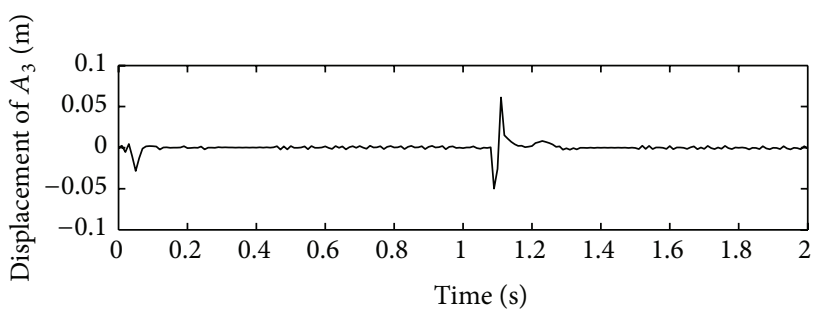

(h)

FIGURE 3: State response of MMAV using SMC based on dynamic inversion.

that the longitudinal displacement of moving mass is constrained within $\pm 0.16 \mathrm{~m}$, and the radical displacements of two moving masses are constrained within $\pm 0.08 \mathrm{~m}$ (The maximum displacement is $\pm 0.05 \mathrm{~m}$ ); just with a minute displacement of moving mass, the MMAV can be controlled, and the control system has favorable dynamic performance to meet the design requirements. The simulation of SMC without fuzzy logic function are carried out in this paper, the displacements of three moving masses in MMAV is shown in Figure 3, it can be seen that the high-frequency chattering appeared in the control signals, this means the three moving masses in MMAV will reciprocate with high frequency, the high-frequency chattering should be restrained with respect to energy saving or extending using life. Correspondingly, Figure 4 shows that the high-frequency chattering disappeared in the control signals and the displacements of three moving masses in MMAV using FSMC. According to the results in the figure, this method can restrain the highfrequency chattering of the system effectively.

\section{Conclusions}

An autopilot for a nonlinear six-degree-of-freedom MMAV is introduced in this paper based on fuzzy sliding mode control, using dynamic inversion techniques. Simulation 


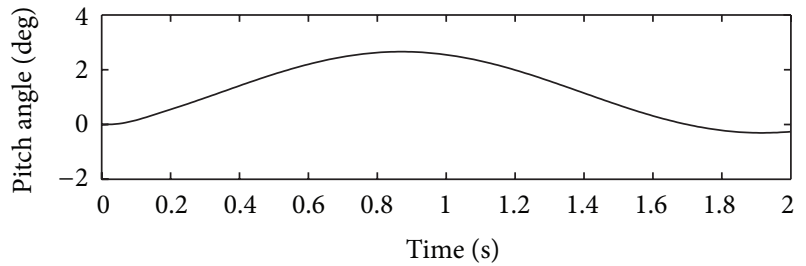

(a)

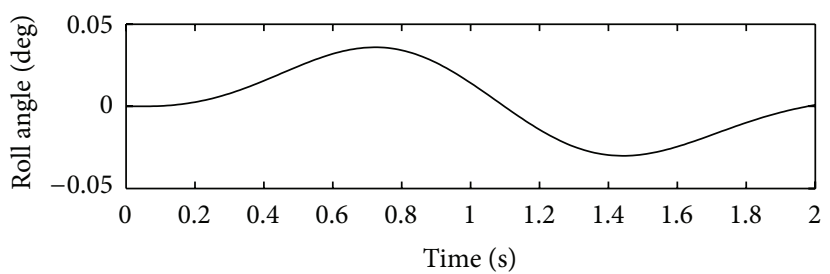

(c)

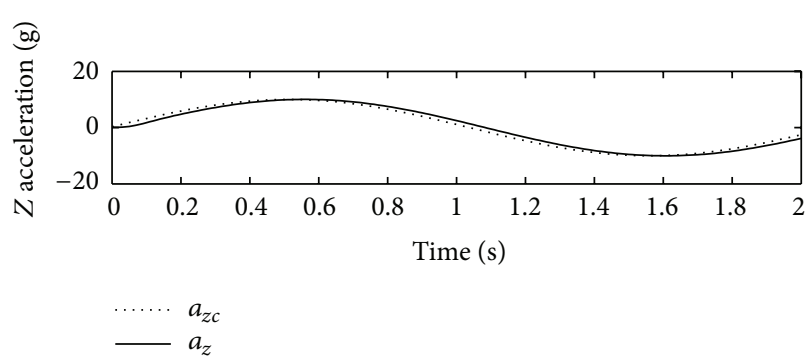

(e)

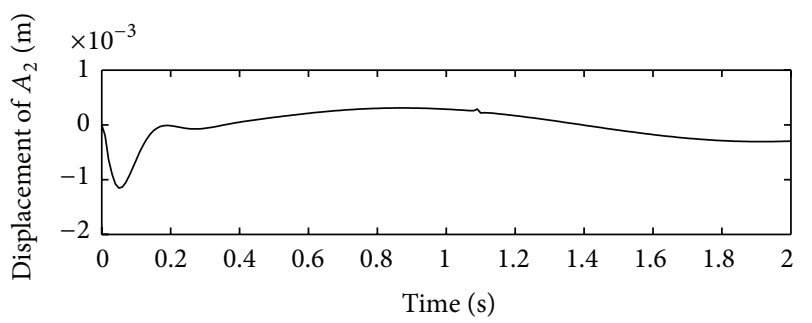

(g)

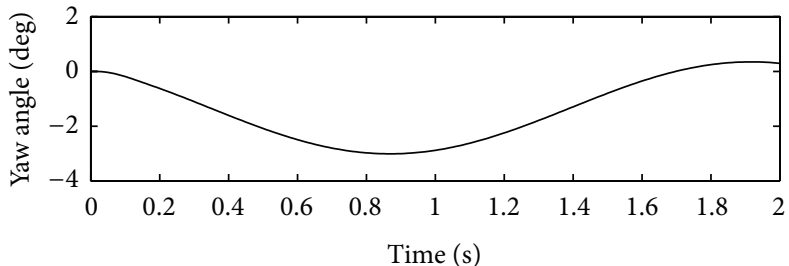

(b)

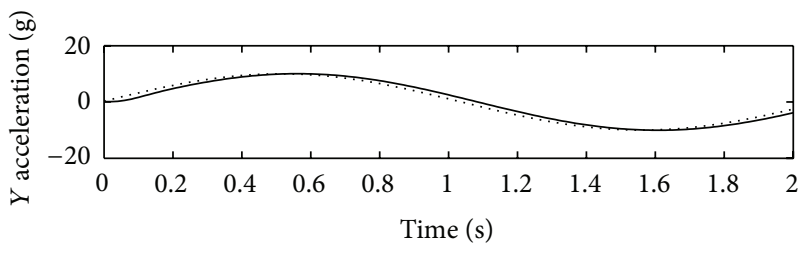

$\begin{array}{ll}\cdots \cdots & a_{y c} \\ - & a_{y}\end{array}$

(d)

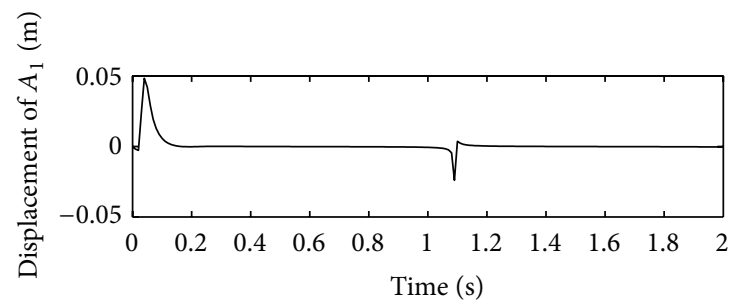

(f)

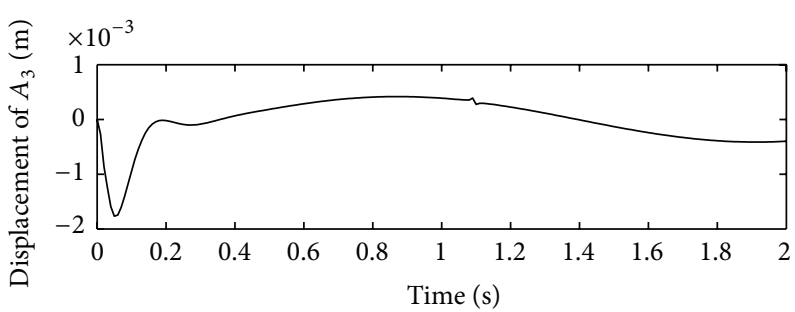

(h)

FIgURE 4: State response of MMAV using FSMC based on dynamic inversion.

results indicate that the resultant control system works well and effectively in MMAV flight control system. Because the stability control mode used by MMAV with three moving masses is three-channel, and the deduced mathematical model is too complicated, the dynamical model of MMAV is still a nonlinear system after reasonable simplification. So, this control system is hard to design. The time-scale separation theory is proposed, in which the dynamical model of MMAV is divided into the dynamics of fast state and the dynamics of slow state, in view to two dynamical subsystems, fuzzy sliding mode control system is designed based on dynamic inversion, this approach can improve the robustness of dynamic inversion effectively in addition to restrain system chattering. The simulation results show that the flight control system of MMAV has good dynamic behavior and strong robustness. As the mechanism of mass moment control is very complicated and the research about that is in the beginning, and the public references are insufficient, we only give some useful discussions about the control system design method of MMAV in this paper.

\section{Conflict of Interests}

The authors declare that there is no conflict of interests regarding the publication of this paper. 


\section{Acknowledgments}

This work is supported by the Natural Science Foundation of Heilongjiang Province of China (F201221), the Training Program of Harbin Engineering University for the National Natural Science Foundation of China and Fundamental Research Funds for the Central Universities of China (HEUCF100417, HEUCF130402, HEUCFX41302).

\section{References}

[1] T. Petsopoulos and F. J. Regan, "A moving-mass roll control system for a fixed-trim reentry vehicle," AIAA Paper 94-0033, pp. 1-11, Reno, Nev, USA, 1994.

[2] R. H. Byrne, B. R. Sturgis, and R. D. Robinett, "A moving mass trim control system for reentry vehicle guidance," Tech. Rep. AIAA-96-3438-CP, pp. 644-650, San Diego, Calif, USA, 1996.

[3] R. D. Robinett III, B. R. Sturgis, and S. A. Kerr, "Moving mass trim control for aerospace vehicles," Journal of Guidance, Control, and Dynamics, vol. 19, no. 5, pp. 1064-1070, 1996.

[4] C. A. Woolsey and N. E. Leonard, "Moving mass control for underwater vehicles," in Proceedings of the American Control Conference (ACC '02), pp. 2824-2829, Anchorage, Alaska, USA, May 2002.

[5] P. K. Menon, S. S. Vaddi, and E. J. Ohlmeyer, "Finite-horizon robust integrated guidance-control of a moving-mass actuated kinetic warhead," Tech. Rep. AIAA 2006-6787, pp. 1-13, Keystone, Colo, USA, 2006.

[6] J.-W. Li, B.-W. Song, and C. Shao, "Tracking control of autonomous underwater vehicles with internal moving mass," Acta Automatica Sinica, vol. 34, no. 10, pp. 1319-1323, 2008.

[7] X.-Y. Zhang, Y.-Z. He, and Z.-C. Wang, "Robust control of mass moment interception missile based on $H_{\infty}$ performance characteristics," Acta Aeronautica et Astronautica Sinica, vol. 28, no. 3, pp. 634-640, 2007.

[8] J. Liu, X. Gao, and Y. Ma, "Study on guidance and control technology of mass moment vehicle," in Proceedings of the IEEE International Conference on Control and Automation (ICCA '09), pp. 679-684, Christchurch, New Zealand, December 2009.

[9] V. I. Utkin, Sliding Modes in Control and Optimization, Communications and Control Engineering Series, Springer, Berlin, Germany, 1992.

[10] L. Wu, H. Gao, and C. Wang, "Quasi sliding mode control of differential linear repetitive processes with unknown input disturbance," IEEE Transactions on Industrial Electronics, vol. 58, no. 7, pp. 3059-3068, 2011.

[11] L. Wu, D. W. C. Ho, and C. W. Li, "Sliding mode control of switched hybrid systems with stochastic perturbation," Systems and Control Letters, vol. 60, no. 8, pp. 531-539, 2011.

[12] L. Wu and D. W. C. Ho, "Sliding mode control of singular stochastic hybrid systems," Automatica, vol. 46, no. 4, pp. 779783, 2010.

[13] L. Wu and J. Lam, "Sliding mode control of switched hybrid systems with time-varying delay," International Journal of Adaptive Control and Signal Processing, vol. 22, no. 10, pp. 909-931, 2008.

[14] L. Wu and W. X. Zheng, "Passivity-based sliding mode control of uncertain singular time-delay systems," Automatica, vol. 45, no. 9, pp. 2120-2127, 2009.

[15] L. Wu, X. Su, and P. Shi, "Sliding mode control with bounded $L_{2}$ gain performance of Markovian jump singular time-delay systems," Automatica, vol. 48, no. 8, pp. 1929-1933, 2012.
[16] J. A. Burton and A. S. I. Zinober, "Continuous approximation of VSC," International Journal of Systems Science, vol. 17, no. 6, pp. 875-885, 1986.

[17] J. J. E. Slotine and W. Li, Applied Nonlinear Control, Prentice Hall, Englewood Cliffs, NJ, USA, 1991.

[18] A. Levant, "Higher-order sliding modes, differentiation and output-feedback control," International Journal of Control, vol. 76, no. 9-10, pp. 924-941, 2003.

[19] A. Levant, "Homogeneity approach to high-order sliding mode design," Automatica, vol. 41, no. 5, pp. 823-830, 2005.

[20] S. Laghrouche, M. Smaoui, F. Plestan, and X. Brun, "Higher order sliding mode control based on optimal approach of an electropneumatic actuator," International Journal of Control, vol. 79, no. 2, pp. 119-131, 2006.

[21] S. Laghrouche, F. Plestan, and A. Glumineau, "Higher-order sliding mode control based on integral sliding mode," Automatica, vol. 43, no. 3, pp. 531-537, 2007.

[22] Y. B. Shtessel, I. A. Shkolnikov, and A. Levant, "Smooth secondorder sliding modes: missile guidance application," Automatica, vol. 43, no. 8, pp. 1470-1476, 2007.

[23] M. Djemai, J.-P. Barbot, and K. K. Busawon, "Designing R-sliding mode control using smooth iterative manifolds," Mediterranean Journal of Measurement and Control, vol. 4, no. 2, pp. 86-93, 2008.

[24] F. Plestan, A. Glumineau, and S. Laghrouche, "A new algorithm for high-order sliding mode control," International Journal of Robust and Nonlinear Control, vol. 18, no. 4-5, pp. 441-453, 2008.

[25] S. Laghrouche, M. Smaoui, F. Plestan, and X. Brun, "Higher order sliding mode control based on optimal approach of an electropneumatic actuator," International Journal of Control, vol. 79, no. 2, pp. 119-131, 2006.

[26] A. Girin and F. Plestan, "A new experimental test bench for a high performance double electropneumatic actuator system," in Proceedings of the American Control Conference (ACC '09), pp. 3488-3493, Saint-Louis, Mo, USA, June 2009.

[27] A. Levant, "Sliding order and sliding accuracy in sliding mode control," International Journal of Control, vol. 58, no. 6, pp. 1247$1263,1993$.

[28] F. Plestan, E. Moulay, A. Glumineau, and T. Cheviron, "Robust output feedback sampling control based on second-order sliding mode," Automatica, vol. 46, no. 6, pp. 1096-1110, 2010.

[29] F. Plestan, Y. Shtessel, V. Brégeault, and A. Poznyak, "New methodologies for adaptive sliding mode control," International Journal of Control, vol. 83, no. 9, pp. 1907-1919, 2010.

[30] H.-T. Yau and C.-L. Chen, "Chattering-free fuzzy sliding-mode control strategy for uncertain chaotic systems," Chaos, Solitons \& Fractals, vol. 30, no. 3, pp. 709-718, 2006.

[31] H. F. Ho, Y. K. Wong, and A. B. Rad, "Adaptive fuzzy sliding mode control with chattering elimination for nonlinear SISO systems," Simulation Modelling Practice and Theory, vol. 17, no. 7, pp. 1199-1210, 2009.

[32] G. Xu, T. Li, X. Zhang, and L. Zhang, "Modeling and motion analysis of a missile based on mass moment control," Journal of Harbin Engineering University, vol. 32, no. 12, pp. 1588-1593, 2011.

[33] K. Peng, K. Y. Lum, E. K. Poh, and D. Li, "Flight control design using hierarchical dynamic inversion and quasi-steady states," Tech. Rep. AIAA 2008-6491, pp. 1-19, Honolulu, Hawaii, USA, 2008.

[34] A. Ishigame, T. Furukawa, S. Kawamoto, and T. Taniguchi, "Sliding mode controller design based on fuzzy inference for 
nonlinear systems," IEEE Transactions on Industrial Electronics, vol. 40, no. 1, pp. 64-70, 1993.

[35] S.-W. Kim and J.-J. Lee, "Design of a fuzzy controller with fuzzy sliding surface," Fuzzy Sets and Systems, vol. 71, no. 3, pp. 359367, 1995.

[36] C. Houck, J. Joines, and M. Kay, The Genetic Algorithm Optimization Toolbox (GAOT) for Matlab 5, 1996.

[37] W. R. Chadwick, "Augmentation of high-altitude maneuver performance of a tailed-controlled missile using lateral thrust," Tech. Rep. AD-A328973, 1995. 


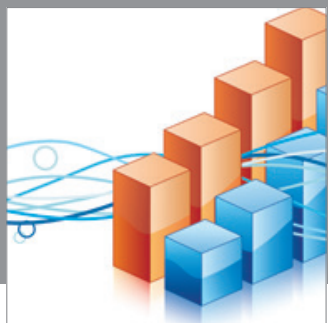

Advances in

Operations Research

mansans

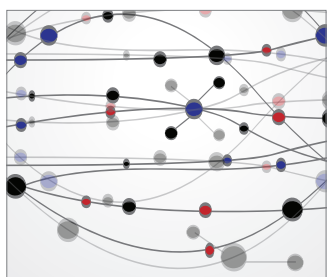

The Scientific World Journal
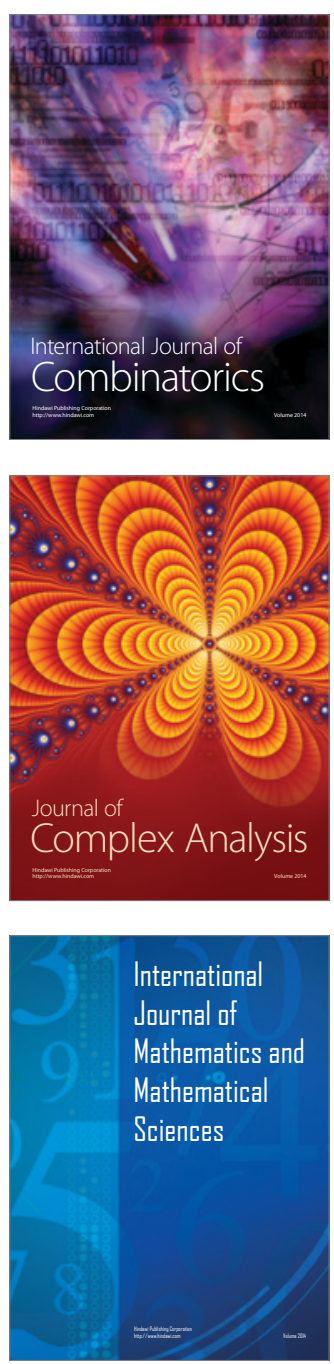
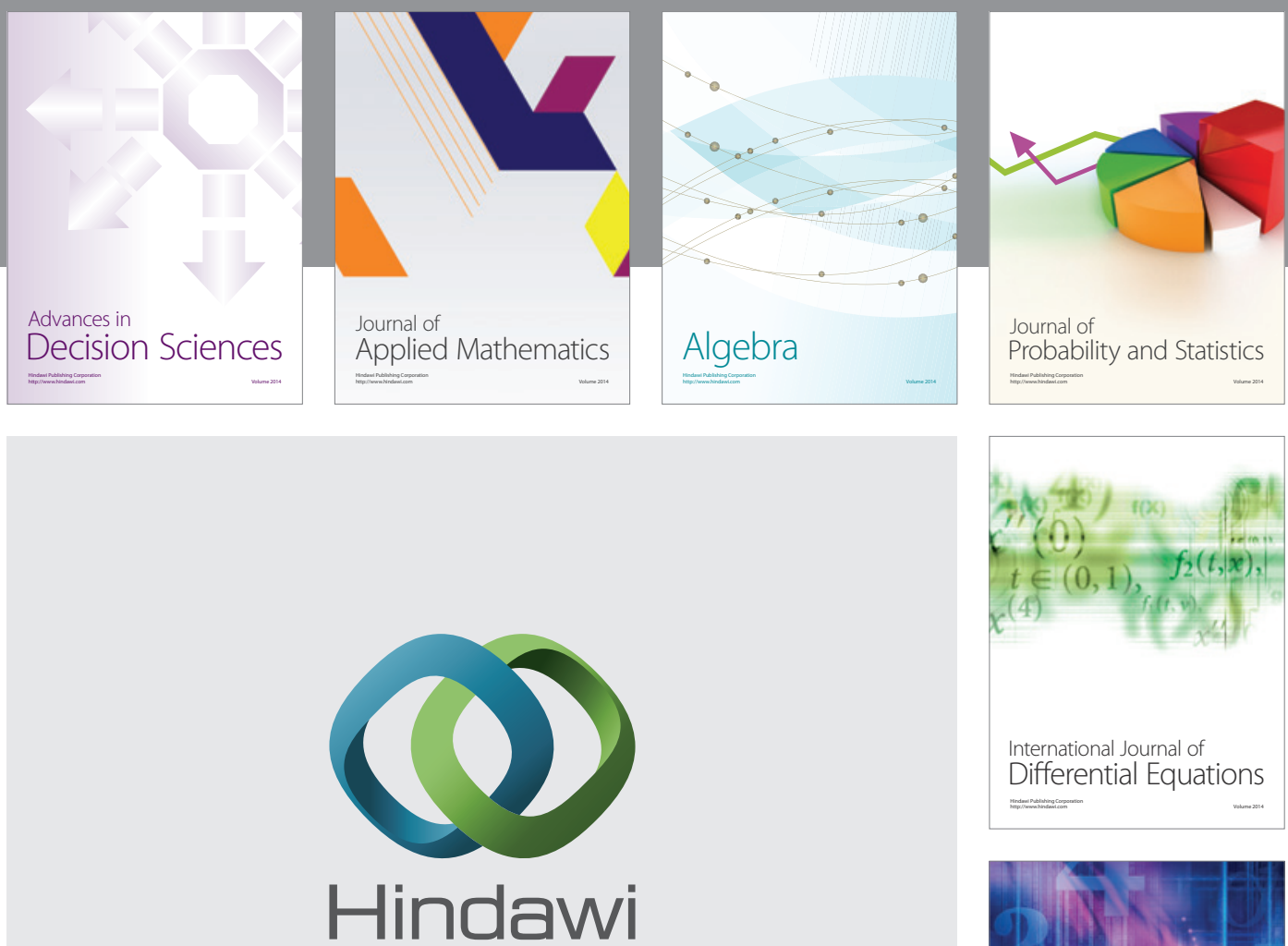

Submit your manuscripts at http://www.hindawi.com
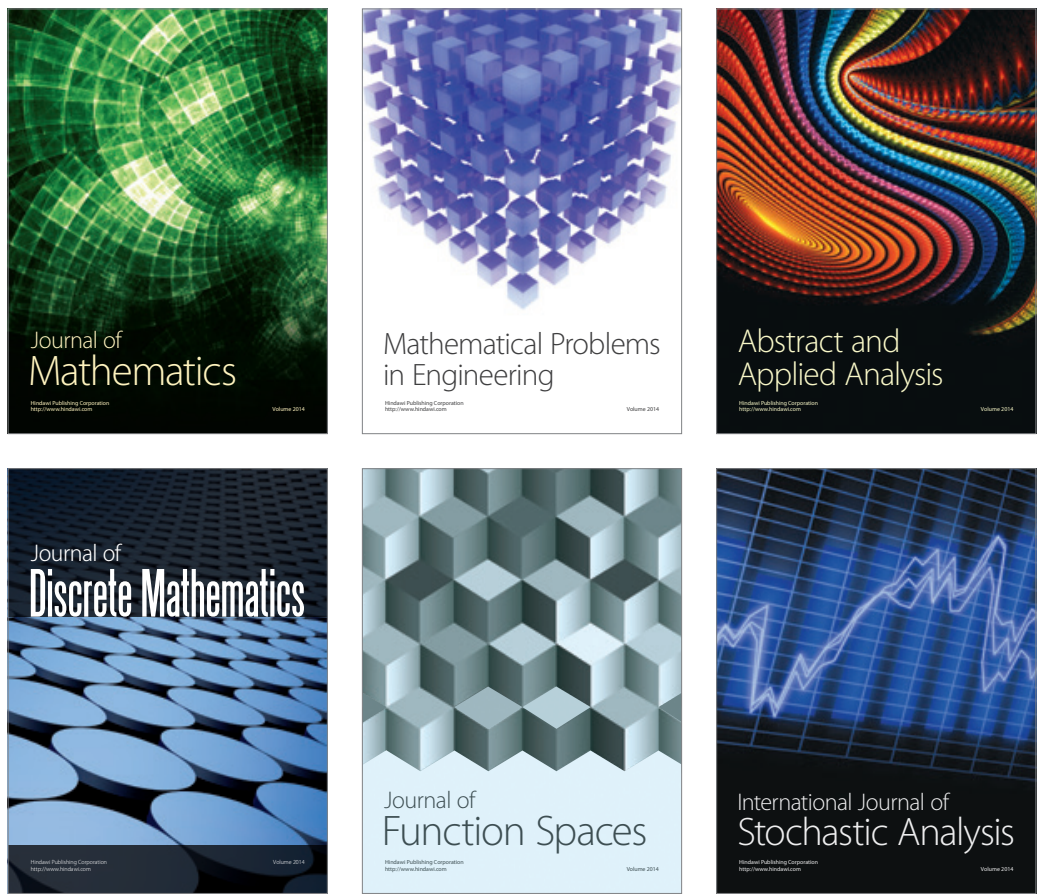

Journal of

Function Spaces

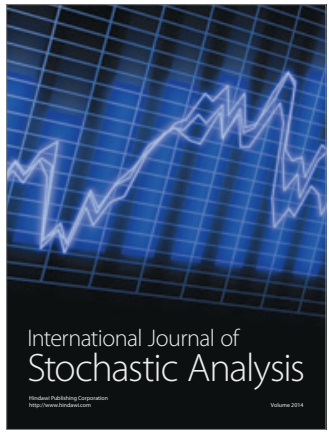

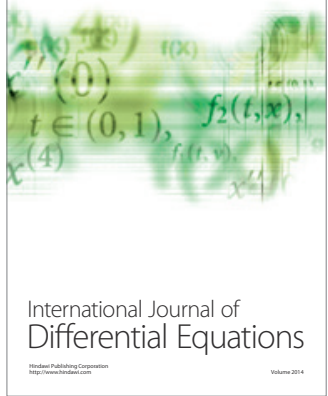
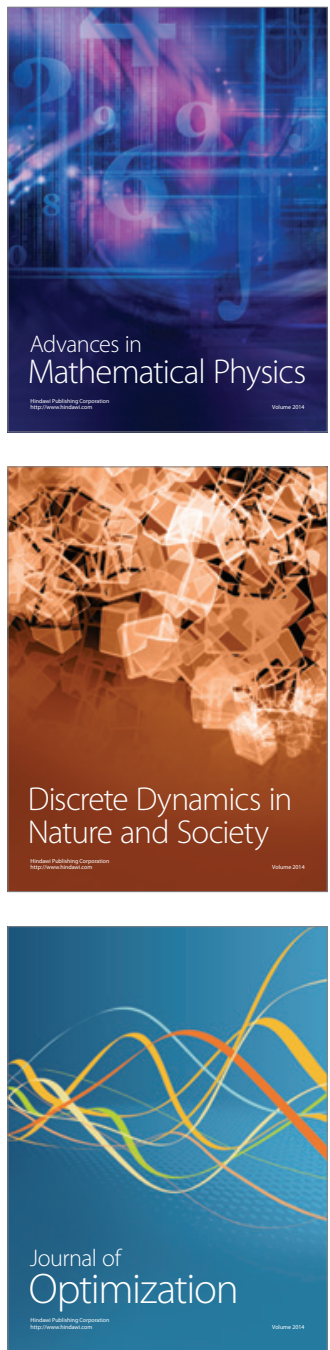